\title{
Emergence of Clade III Candida auris of African Origin in Los Angeles
}

Travis K. Price ${ }^{1}$, Ruel Mirasol ${ }^{1}$, Kevin W. Ward ${ }^{1}$, Ayrton J. Dayo ${ }^{1}$, Sukantha

Chandrasekaran ${ }^{1}$, Omai B. Garner ${ }^{1}$, Annabelle de St Maurice ${ }^{2,3}$ and Shangxin Yang ${ }^{1}$

${ }^{1}$ Department of Pathology and Laboratory Medicine, ${ }^{2}$ Department of Clinical

Epidemiology and Infection Prevention, and ${ }^{3}$ Division of Pediatric Infectious Diseases,

Department of Pediatrics, David Geffen School of Medicine at UCLA, Los Angeles, CA

CORRESPONDING AUTHOR: Shangxin Yang, PhD, D(ABMM); UCLA Clinical

Microbiology Laboratory, 11633 San Vicente Blvd, Los Angeles, CA 90049;

shangxinyang@mednet.ucla.edu

RUNNING TITLE: Clade III Candida auris Outbreaks in Los Angeles

KEY WORDS: Candida auris, Clade III, Los Angeles, Outbreak, Genomic

Characterization

\section{ABSTRACT:}

Candida auris is an emerging multi-drug resistant yeast responsible for invasive infections among hospitalized patients. We described a case series of $C$. auris isolated in the Los Angeles area from an ongoing outbreak. Genomic analysis showed a single Clade III lineage of African origin in all 6 cases since 2019. 


\section{MANUSCRIPT:}

\section{Introduction}

Candida auris was first isolated in 2009 from the external ear canal of a 70-yearold patient in Tokyo, Japan ${ }^{1}$. In the decade that followed, cases of $C$. auris bloodstream and other invasive infections have been reported worldwide $\begin{array}{llllll}2 & 3 & 4 & 5 & 6 & 7\end{array}$. Many strains of C. auris are multidrug resistant and in some cases have elevated minimum inhibitory concentrations (MICs) to all three classes of antifungals including azoles, echinocandins and polyenes. The Centers for Disease Control and Prevention (CDC) recently listed $C$. auris as one of five "urgent threats" to public health in the 2019 Antibiotic Resistance (AR) Threats Report ${ }^{8}$, highlighting the need for active surveillance and appropriate infection prevention.

Whole genome sequencing (WGS) and phylogenetic analyses revealed the presence of at least four major clades of $C$. auris with worldwide geographic associations $^{9}{ }^{10}$. In the United States, outbreaks have been documented in New York, New Jersey, and Illinois; these were generally caused by strains of $C$. auris that were part of Clades I and IV ${ }^{11}$.

Here, we describe several cases of $C$. auris colonization and infection in patients of long-term acute care (LTAC) facilities in the Los Angeles area. WGS revealed that all isolates were part of Clade III, likely representing a unique outbreak in the United States. This is the first description of $C$. auris strains in Southern California.

\section{Methods}


A laboratory-developed polymerase chain reaction (PCR) test implemented by the clinical microbiology laboratory in September 2019, was used for the detection of $C$. auris from inguinal-axillary swabs and yeast isolates from positive fungal culture of clinical specimens as a means to actively screen high-risk patients transferred to UCLA from LTAC facilities and skilled nursing facilities (SNFs). Nucleic acid extraction using the EZ1 Tissue Extraction kit (Qiagen, Germantown, MD) was performed followed by PCR using the DiaSorin Molecular LIAISON ${ }^{\circledR}$ MDX platform (DiaSorin Molecular, Cypress, CA). PCR-positive swab specimens were cultured; yeast isolates were identified using VITEK MS MALDI-TOF system (bioMérieux Inc, Hazelwood, MO).

Antifungal susceptibility testing by broth microdilution was performed on panels prepared in-house according to Clinical and Laboratory Standards Institute (CLSI) standards. The CDC tentative MIC breakpoints were used for interpretation of the following drugs: amphotericin B $(\geq 2 \mu \mathrm{g} / \mathrm{ml})$, fluconazole $(\geq 32 \mu \mathrm{g} / \mathrm{ml})$, anidulafungin $(\geq 4 \mu \mathrm{g} / \mathrm{ml})$, caspofungin $(\geq 2 \mu \mathrm{g} / \mathrm{ml})$, micafungin $(\geq 4 \mu \mathrm{g} / \mathrm{ml})$. Voriconazole, itraconazole, and posaconazole were also tested but no interpretative criteria for $C$. auris are available.

WGS was performed using Illumina MiSeq (Illumina, San Diego, CA). Analyses were performed using CLC Genomics Workbench (Qiagen, Valencia, CA, USA) and Geneious Prime (Biomatters, New Zealand). Reads were mapped to a reference $C$. auris complete genome (ATCC MYA-5002, NCBI: CP043531); this strain is part of Clade III. Sequences were compared by K-mer analysis and single nucleotide polymorphism (SNP) analysis using maximum-likelihood phylogenies. 
This is a retrospective analysis and is part of an infection prevention and quality improvement project. The institutional review boards (IRB) approval was waived by the UCLA Office of the Human Research Protection Program.

\section{Results}

In one year (09/2019 - 09/2020), we screened 113 patients using our in-house C. auris PCR assay; six patients have tested positive for $C$. auris. Cycle threshold (Ct) values ranged from 22.6 to 39.7 (Table 1). Of the six PCR-positive patients, herein referred to as patients A through F, five tested positive between July and September 2020 (patients B-F); while patient A tested positive in October 2019.

All six patients were residents of 4 different LTAC facilities within the Los Angeles county area; all had history of tracheostomy. Patients $\mathrm{A}$ and $\mathrm{F}$ had prior history of $C$. auris colonization. The microbiological findings of each patient are described in Table 1. C. auris isolates grew from inguinal-axillary swabs of $4 / 6$ patients $(A, C, D, E)$ and from other clinical specimens of patients A (tracheal aspirate) and F (pleural fluid). We did not isolate $C$. auris from patient $\mathrm{B}(\mathrm{Ct}=39.5)$.

All C. auris isolates were resistant to amphotericin $\mathrm{B}(\mathrm{MIC}=2 \mu \mathrm{g} / \mathrm{ml})$ and fluconazole (MIC>64 $\mathrm{g} / \mathrm{ml}$ ) but susceptible to echinocandins (Table 2).

K-mer analysis was performed using 252 publicly available sequences of $C$. auris described previously ${ }^{12}$ (Supplemental Data 1) in addition to the sequences of the six isolates described here. All UCLA isolates belonged to Clade III (Supplemental Data 1). In this analysis, Clade III contained 56 isolates; these were primarily isolated from the African continent $(n=44)^{11}$ (Supplemental Figure 1). 
Isolates from all four clades have been identified in the US and show a geographic relationship ${ }^{11}$; Clade I is predominant. A k-mer phylogenetic analysis of 57 C. auris isolated in the US was performed (Figure 1). Clade III $(n=7)$, which included the six UCLA isolates, had one additional isolate which originated from Indiana and was isolated in March of 2017 (Genbank accession \# SRR7909359). SNP analysis showed that the UCLA isolates were closely related to one another (3-78 SNPs) as well as to the Indiana isolate (66 - 138 SNPs) (Supplemental Table 1).

To further characterize the $C$. auris isolates, we analyzed the sequence of two genes associated with antifungal resistance: erg11, which encodes lanosterol 14-alpha demethylase, and $f k s 1$, a subunit of 1,3- $\beta-D$-glucan synthase. Sequences of erg11 were identical for all UCLA isolates; all had a 99.6\% pairwise nucleotide identity to the reference (CP043531) and two amino acid substitutions: V125A and F126L (Supplemental Table 2). Mutations at amino acid 126 are associated with increased azole resistance in $C$. auris ${ }^{9}$ and are a common feature of Clade III isolates ${ }^{13}$. The specific F126L mutation appears to be exclusive to Clade III $^{12}$. These findings are consistent with the antifungal susceptibility testing which showed that all isolates were resistant to fluconazole (Table 2). Sequences of fks 1 were identical in five of the UCLA isolates (A1, A2, C1, D1, E1) and showed 99.9\% pairwise nucleotide identity to the reference (CP043531); these isolates had one amino acid substitution: 11572L (Supplemental Table 2). Isolate UCLA_F1 had the same substitution in addition to I1095L. All isolates had a WT serine at amino acid 639; mutations at position 639 are linked to echinocandin resistance in C. auris ${ }^{14}$. All isolates were susceptible to caspofungin, micafungin and anidulafungin. 


\section{Discussion}

Here, our use of an in-house PCR test to actively screen patients for $C$. auris colonization has proven to be vital in identifying and preventing the spread of this pathogen in our hospital system. WGS of isolates obtained from several patients of LTAC facilities revealed two important findings. First, these isolates are closely related to one another; this confirms the existence of an ongoing outbreak with community spread in the Los Angeles area. Second, these isolates are part of Clade III of African origin; this suggests a unique introduction of $C$. auris into the United States because this clade has not been implicated in outbreaks in other states.

The isolates described here were all resistant to fluconazole and Amphotericin B but susceptible to echinocandins. Additionally, all isolates had an F126L mutation in the erg11 gene, which is associated with fluconazole resistance and unique to Clade III strains of $C$. auris.

Limitations of this study include the lack of any additional epidemiological history of the patients related to exposures including travel. Being able to link patients to a country with known $C$. auris outbreaks of Clade III strains would be essential to understanding how the current Los Angeles outbreak began.

In conclusion, we have identified a unique Clade III $C$. auris strain in an ongoing outbreak in the LTAC facilities since 2019 , indicating active community spread of multidrug resistant $C$. auris in the Los Angeles area. 


\section{FIGURES \& TABLES}

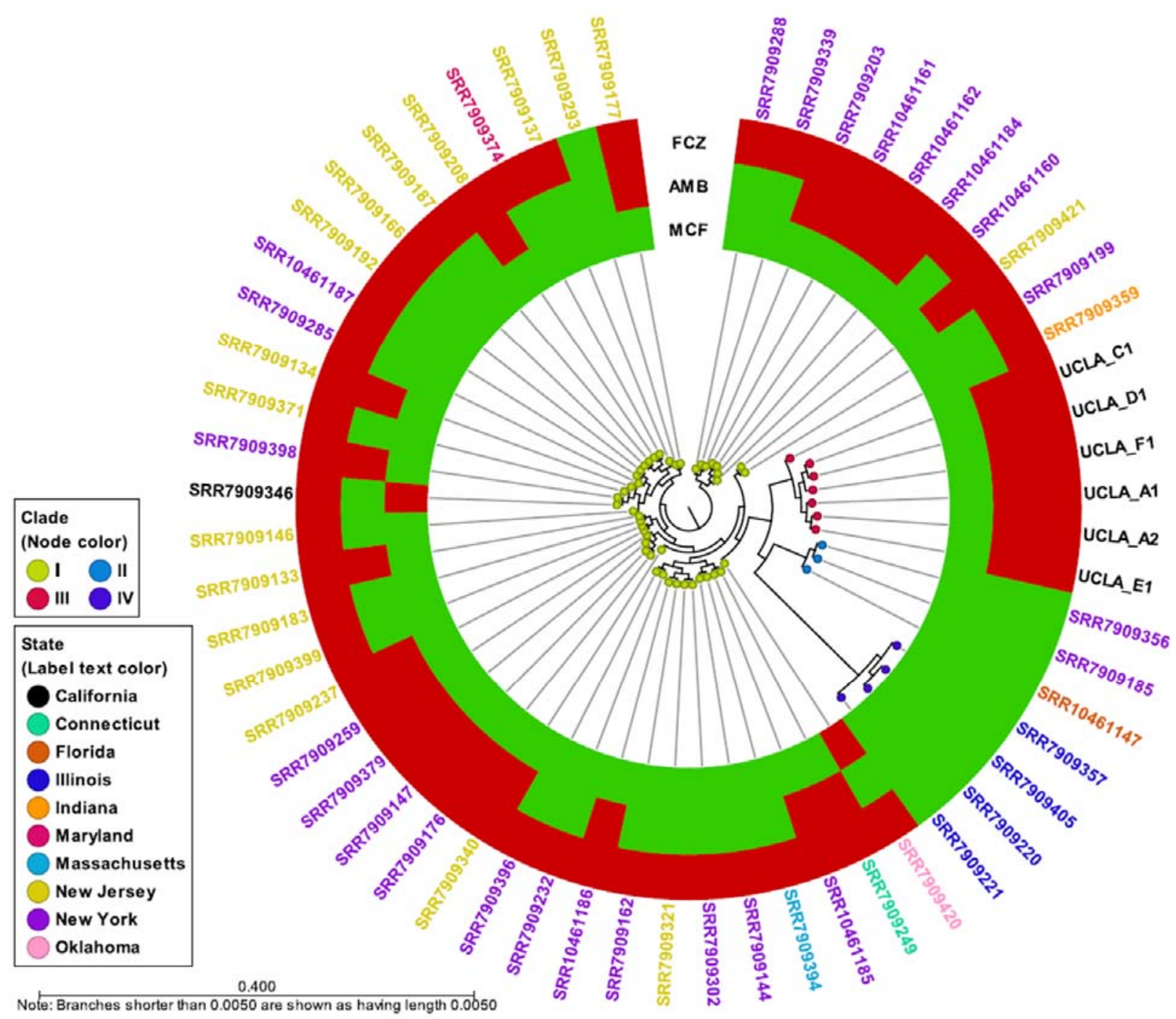

Figure 1. K-mer Analysis of 57 C. auris Isolates from the United States. K-mer analysis was performed using CLC Genomics Workbench (Qiagen, Valencia, CA, USA) using genome sequences from 52 publicly available $C$. auris strains on NCBI GenBank as well as sequences from UCLA_A1, UCLA_A2, UCLA_C1, UCLA_D1, UCLA_E1, and UCLA_F1. A list of sequences used is found in Supplemental Data 1. Each node represents a unique isolate; the node color refers to the clade. The color of the isolate name (i.e., label text color) refers to the state of origin. The metadata shows the susceptibility of each isolate to fluconazole (FCZ), amphotericin B (AMB), and micafungin (MCF); red indicates resistant, green indicates susceptible. 
Table 1. Patient test results, clinical history, and $C$. auris isolate information.

\begin{tabular}{|c|c|c|c|}
\hline Patient & $\begin{array}{c}\text { Month of } \\
\text { Positive PCR }\end{array}$ & $\begin{array}{c}\text { PCR Ct } \\
\text { Value }\end{array}$ & $\begin{array}{c}\text { C. auris Isolate } \\
\text { (Specimen Type) }\end{array}$ \\
\hline A & $10 / 2019$ & 22.6 & $\begin{array}{l}\text { UCLA_A1 (Inguinal-Axillary) } \\
\text { UCLA_A2 (Tracheal) }\end{array}$ \\
\hline B & $7 / 2020$ & 39.5 & Not isolated \\
\hline C & $8 / 2020$ & 22.6 & UCLA_C1 (Inguinal-Axillary) \\
\hline D & $8 / 2020$ & 39.7 & UCLA_D1 (Inguinal-Axillary) \\
\hline E & $8 / 2020$ & 28.3 & UCLA_E1 (Inguinal-Axillary) \\
\hline F & $9 / 2020$ & 30.6 & UCLA_F1 (Pleural Fluid, Rt) \\
\hline
\end{tabular}

${ }^{a} C$. auris was not isolated from the inguinal-axillary surveillance swab of patient $B$

Table 2. Antifungal susceptibility results for $C$. auris isolates.

\begin{tabular}{|l|c|c|c|c|c|c|}
\hline \multicolumn{7}{|c|}{ MIC $(\boldsymbol{\mu g} / \mathbf{m l}$ ) (interpretation) for $\boldsymbol{C}$. auris isolate: } \\
\hline Antifungal & UCLA_A1 & UCLA_A2 & UCLA_C1 & UCLA_D1 & UCLA_E1 & UCLA_F1 \\
\hline Amphotericin B & $2(\mathrm{R})$ & $2(\mathrm{R})$ & $2(\mathrm{R})$ & $2(\mathrm{R})$ & $2(\mathrm{R})$ & $2(\mathrm{R})$ \\
\hline Fluconazole & $>64(\mathrm{R})$ & $>64(\mathrm{R})$ & $>64(\mathrm{R})$ & $>64(\mathrm{R})$ & $>64(\mathrm{R})$ & $>64(\mathrm{R})$ \\
\hline Voriconazole & 2 & 1 & 1 & 0.5 & 0.5 & 2 \\
\hline Itraconazole & 1 & 1 & 0.5 & 0.25 & 0.25 & 1 \\
\hline Posaconazole & 0.06 & 0.12 & $\leq 0.03$ & $\leq 0.03$ & 0.06 & 0.06 \\
\hline Anidulafungin & $0.5(\mathrm{~S})$ & $0.12(\mathrm{~S})$ & $0.25(\mathrm{~S})$ & $0.12(\mathrm{~S})$ & $0.06(\mathrm{~S})$ & $1(\mathrm{~S})$ \\
\hline Caspofungin & $0.5(\mathrm{~S})$ & $0.12(\mathrm{~S})$ & $0.5(\mathrm{~S})$ & $0.5(\mathrm{~S})$ & $0.12(\mathrm{~S})$ & $0.5(\mathrm{~S})$ \\
\hline Micafungin & $0.25(\mathrm{~S})$ & $0.25(\mathrm{~S})$ & $0.25(\mathrm{~S})$ & $0.12(\mathrm{~S})$ & $0.25(\mathrm{~S})$ & $0.25(\mathrm{~S})$ \\
\hline
\end{tabular}

MIC testing was performed on panels prepared in-house in accordance with CLSI guidelines. Interpretive breakpoints were defined by the CDC Antifungal Susceptibility Testing and Interpretation guidelines for $C$. auris, which were adapted from interpretive criteria for closely related Candida spp. Tentative breakpoints for the following antifungals were used: amphotericin B $(\geq 2 \mu \mathrm{g} / \mathrm{ml})$, fluconazole $(\geq 32 \mu \mathrm{g} / \mathrm{ml})$, anidulafungin $(\geq 4 \mu \mathrm{g} / \mathrm{ml})$, caspofungin $(\geq 2 \mu \mathrm{g} / \mathrm{ml})$, micafungin $(\geq 4 \mu \mathrm{g} / \mathrm{ml})$. S=susceptible, l=intermediate, $R=$ resistant. 


\section{SUPPLEMENTAL FIGURES \& TABLES}

\section{Supplemental Table 1. SNP analysis of $C$. auris isolates.}

\begin{tabular}{|c|c|c|c|c|c|c|c|c|c|c|}
\hline & 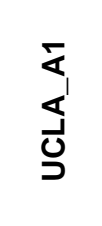 & 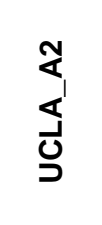 & $\begin{array}{l}\bar{U} \\
\bar{J} \\
\bar{J}\end{array}$ & $\begin{array}{l}\overline{0} \\
\bar{J} \\
\bar{\jmath}\end{array}$ & 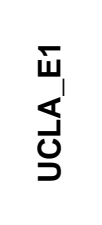 & 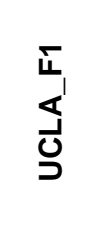 & 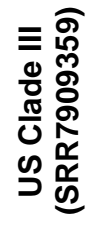 & 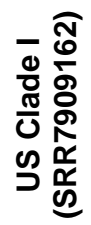 & 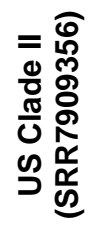 & 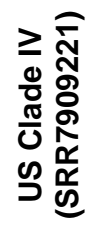 \\
\hline UCLA_A1 & --- & 3 & 12 & 10 & 7 & 76 & 66 & 24373 & 35804 & 95894 \\
\hline UCLA_A2 & 3 & --- & 11 & 9 & 6 & 77 & 65 & 24372 & 35803 & 95893 \\
\hline UCLA_C1 & 12 & 11 & --- & 4 & 7 & 76 & 74 & 24381 & 35808 & 95896 \\
\hline UCLA_D1 & 10 & 9 & 4 & --- & 5 & 78 & 72 & 24379 & 35810 & 95897 \\
\hline UCLA_E1 & 7 & 6 & 7 & 5 & --- & 75 & 69 & 24376 & 35807 & 95897 \\
\hline UCLA_F1 & 76 & 77 & 76 & 78 & 75 & --- & 138 & 24402 & 35796 & 95919 \\
\hline $\begin{array}{c}\text { US Clade III } \\
\text { (SRR7909359) }\end{array}$ & 66 & 65 & 74 & 72 & 69 & 138 & --- & 24384 & 35814 & 95909 \\
\hline $\begin{array}{c}\text { US Clade I } \\
\text { (SRR7909162) }\end{array}$ & 24373 & 24372 & 24381 & 24379 & 24376 & 24402 & 24384 & --- & 36609 & 97646 \\
\hline $\begin{array}{c}\text { US Clade II } \\
\text { (SRR7909356) }\end{array}$ & 35804 & 35803 & 35808 & 35810 & 35807 & 35796 & 35814 & 36609 & --- & 95704 \\
\hline $\begin{array}{c}\text { US Clade IV } \\
\text { (SRR7909221) }\end{array}$ & 95894 & 95893 & 95896 & 95897 & 95897 & 95919 & 95909 & 95704 & 97646 & --- \\
\hline
\end{tabular}

Reads were mapped to the $C$. auris reference genome (CP043531), consensus sequences were extracted from the alignments and SNP analysis was performed.

\section{Supplemental Table 2. Nucleotide and amino acid variants of $C$. auris isolates}

\begin{tabular}{|c|c|c|c|c|}
\hline \multirow[b]{2}{*}{$\begin{array}{l}\text { C. auris } \\
\text { Isolate }\end{array}$} & \multicolumn{2}{|c|}{ Erg11 } & \multicolumn{2}{|c|}{ Fks1 } \\
\hline & $\begin{array}{l}\text { Nucleotide } \\
\text { Pairwise Identity }\end{array}$ & $\begin{array}{l}\text { Protein Sequence } \\
\text { Variants }\end{array}$ & $\begin{array}{l}\text { Nucleotide } \\
\text { Pairwise Identity }\end{array}$ & $\begin{array}{l}\text { Protein Sequence } \\
\text { Variants }\end{array}$ \\
\hline UCLA_A1 & $99.6 \%$ & $\begin{array}{l}\text { p.V125A } \\
\text { p.F126L }\end{array}$ & $99.9 \%$ & p.I1572L \\
\hline UCLA_A2 & $99.6 \%$ & $\begin{array}{l}\text { p.V125A } \\
\text { p.F126L }\end{array}$ & $99.9 \%$ & p.I1572L \\
\hline UCLA_C1 & $99.6 \%$ & $\begin{array}{l}\text { p.V125A } \\
\text { p.F126L }\end{array}$ & $99.9 \%$ & p.I1572L \\
\hline UCLA_D1 & $99.6 \%$ & $\begin{array}{l}\text { p.V125A } \\
\text { p.F126L }\end{array}$ & $99.9 \%$ & p.I1572L \\
\hline UCLA_E1 & $99.6 \%$ & $\begin{array}{l}\text { p.V125A } \\
\text { p.F126L }\end{array}$ & $99.9 \%$ & p.I1572L \\
\hline UCLA_F1 & $99.6 \%$ & $\begin{array}{l}\text { p.V125A } \\
\text { p.F126L }\end{array}$ & $99.8 \%$ & $\begin{array}{l}\text { p.I1095L } \\
\text { p.I1572L }\end{array}$ \\
\hline
\end{tabular}

Nucleotide pairwise identity to C. auris strain ATCC MYA-5002 (CP043531), and protein translation and variant analysis was performed using Geneious Prime (Biomatters, New Zealand). 


\section{Country (Metadata layer \#1) \\ Australia \\ Canada \\ Kenya \\ South Africa \\ Spain \\ United States}

0.020

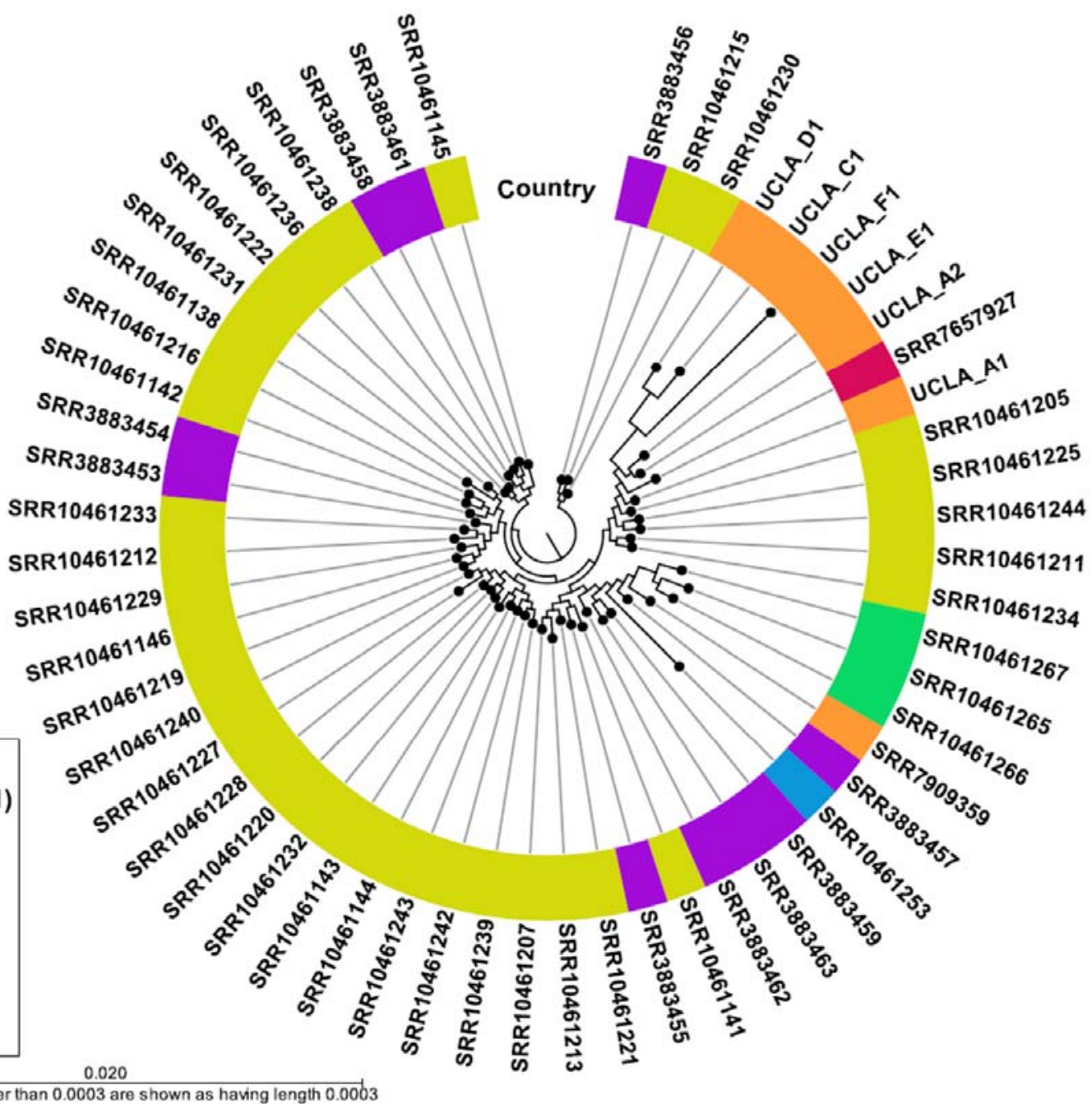

Supplemental Figure 1. K-mer Analysis of 56 C. auris Isolates in Clade III. K-mer analysis was performed using CLC Genomics Workbench (Qiagen, Valencia, CA, USA) using genome sequences from 50 publicly available $C$. auris strains on NCBI GenBank as well as sequences from UCLA_A1, UCLA_A2, UCLA_C1, UCLA_D1, UCLA_E1, and UCLA_F1. A list of sequences used is found in Supplemental Data 1. The metadata (i.e., metadata layer \#1) shows the country of origin. 


\section{REFERENCES}

1. Satoh K, Makimura K, Hasumi Y, Nishiyama Y, Uchida K, Yamaguchi H. Candida auris sp. nov., a novel ascomycetous yeast isolated from the external ear canal of an inpatient in a japanese hospital. Microbiol Immunol. 2009;53(1):41-44. doi:

10.1111/j.1348-0421.2008.00083.x [doi].

2. Calvo B, Melo AS, Perozo-Mena A, et al. First report of candida auris in america:

Clinical and microbiological aspects of 18 episodes of candidemia. $J$ Infect.

2016;73(4):369-374. doi: 10.1016/j.jinf.2016.07.008 [doi].

3. Belkin A, Gazit Z, Keller N, et al. Candida auris infection leading to nosocomial transmission, israel, 2017. Emerg Infect Dis. 2018;24(4):801-804. doi: 10.3201/eid2404.171715 [doi].

4. Ruiz-Gaitan A, Moret AM, Tasias-Pitarch M, et al. An outbreak due to candida auris with prolonged colonisation and candidaemia in a tertiary care european hospital. Mycoses. 2018;61(7):498-505. doi: 10.1111/myc.12781 [doi].

5. Armstrong PA, Rivera SM, Escandon P, et al. Hospital-associated multicenter outbreak of emerging fungus candida auris, colombia, 2016. Emerg Infect Dis. 2019;25(7):10.3201/eid2507.180491. doi: 10.3201/eid2507.180491 [doi].

6. Rhodes J, Abdolrasouli A, Farrer RA, et al. Genomic epidemiology of the UK outbreak of the emerging human fungal pathogen candida auris. Emerg Microbes Infect. 2018;7(1):43-x. doi: 10.1038/s41426-018-0045-x [doi]. 
7. Tsay S, Welsh RM, Adams EH, et al. Notes from the field: Ongoing transmission of candida auris in health care facilities - united states, june 2016-may 2017. MMWR Morb Mortal Wkly Rep. 2017;66(19):514-515. doi: 10.15585/mmwr.mm6619a7 [doi].

8. CDC. Antibiotic resistance threats in the united states, 2019. . 2019.

9. Lockhart SR, Etienne KA, Vallabhaneni S, et al. Simultaneous emergence of multidrug-resistant candida auris on 3 continents confirmed by whole-genome sequencing and epidemiological analyses. Clin Infect Dis. 2017;64(2):134-140. doi: 10.1093/cid/ciw691 [doi].

10. Chow NA, de Groot T, Badali H, Abastabar M, Chiller TM, Meis JF. Potential fifth clade of candida auris, iran, 2018. Emerg Infect Dis. 2019;25(9):1780-1781. doi: 10.3201/eid2509.190686 [doi].

11. Chow NA, Gade L, Tsay SV, et al. Multiple introductions and subsequent transmission of multidrug-resistant candida auris in the USA: A molecular epidemiological survey. Lancet Infect Dis. 2018;18(12):1377-1384. doi: S14733099(18)30597-8 [pii].

12. Chow NA, Munoz JF, Gade L, et al. Tracing the evolutionary history and global expansion of candida auris using population genomic analyses. mBio. 2020;11(2):10.1128/mBio.03364-19. doi: e03364-19 [pii].

13. Healey KR, Kordalewska M, Jimenez Ortigosa C, et al. Limited ERG11 mutations identified in isolates of candida auris directly contribute to reduced azole susceptibility. 
medRxiv preprint doi: https://doi.org/10.1101/2020.10.26.20214908; this version posted October 27, 2020. The copyright holder for this preprint (which was not certified by peer review) is the author/funder, who has granted medRxiv a license to display the preprint in perpetuity.

All rights reserved. No reuse allowed without permission.

Antimicrob Agents Chemother. 2018;62(10):10.1128/AAC.01427-18. Print 2018 Oct. doi: e01427-18 [pii].

14. Chowdhary A, Prakash A, Sharma C, et al. A multicentre study of antifungal susceptibility patterns among 350 candida auris isolates (2009-17) in india: Role of the ERG11 and FKS1 genes in azole and echinocandin resistance. $J$ Antimicrob Chemother. 2018;73(4):891-899. doi: 10.1093/jac/dkx480 [doi]. 